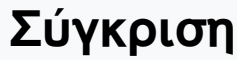

Tóp. 9 (1998)

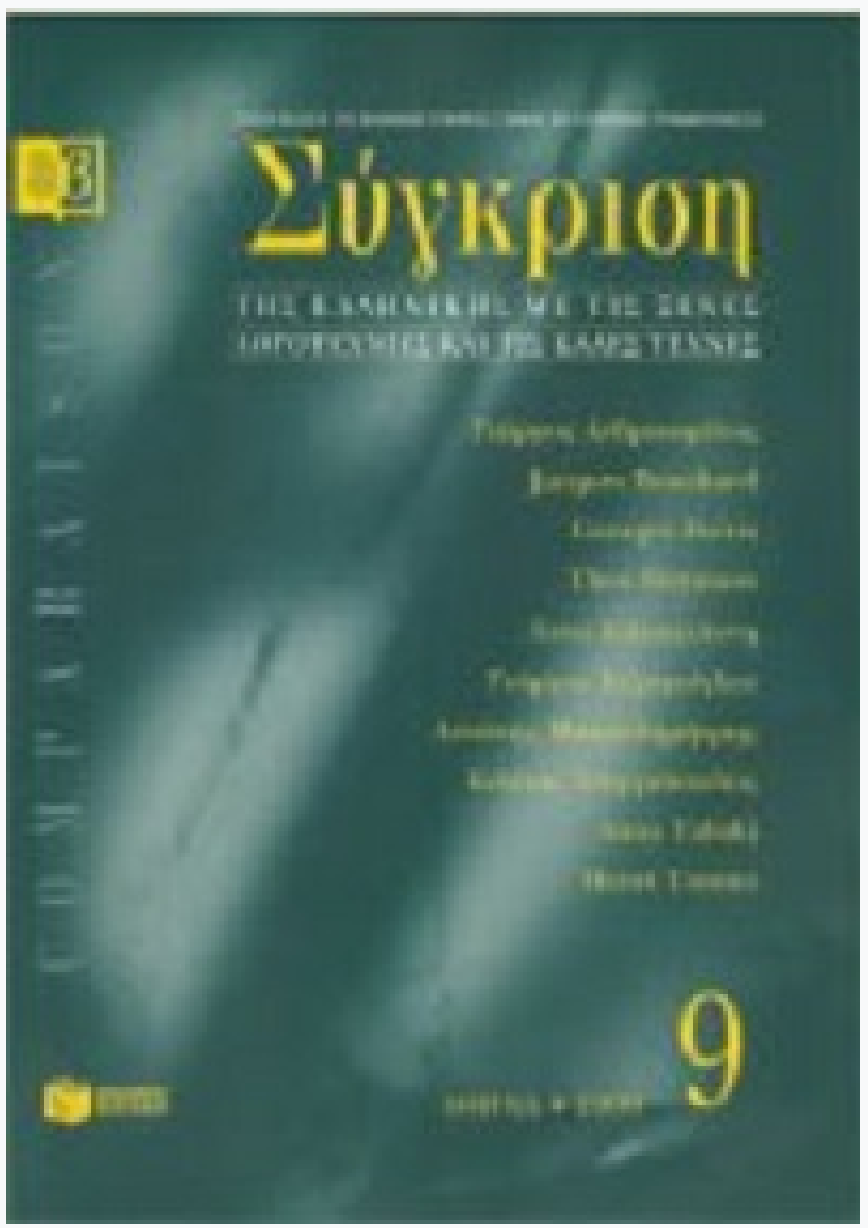

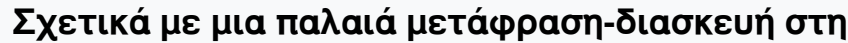 véa $\varepsilon \lambda \lambda \eta v ı$ ń tou Lous-Sebastian Mercier}

\section{Henri Tonnet}

doi: $10.12681 /$ comparison.11449

\section{Copyright $@ 2017$, Henri Tonnet}

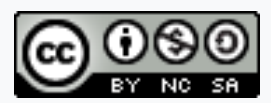

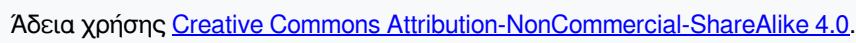

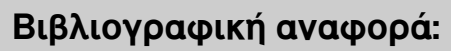

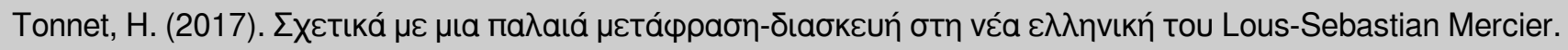

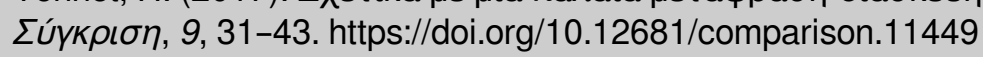




\section{$\grave{A}$ propos d'une ancienne traduction-adaptation de Louis-Sébastien Mercier en grec moderne}

\section{Introduction}

On a depuis longtemps reconnu le rôle essentiel des traductions dans la diffusion des Lumières en Grèce ${ }^{1}$. La critique s'est cependant contentée le plus souvent de relever l'existence des traductions ${ }^{2}$ sans aller plus avant dans la confrontation de ces textes grecs avec leurs modèles français ${ }^{3}$. Et pourtant l'étude attentive des anciennes traductions est riche d'enseignements, en particulier sur l'état de la langue grecque écrite au moment où ces textes ont été publiés. C'est en effet souvent dans sa confrontation avec les grands textes européens, essentiellement français, que la langue grecque moderne a dû se forger de nouveaux termes qui, d'abord liés à une situation concrète de traduction, ont par la suite poursuivi librement leur vie dans le lexique. ${ }^{1}$

Entre toutes les périodes où l'on a fait des traductions en $\operatorname{grec}^{5}$, c'est certainement le XVIIIle siècle qui paraît se prêter le mieux à ce genre d'études ${ }^{6}$. C' est en effet le moment où les Grecs abandonnent résolument la libre adaptation, souvent en vers, pour tenter de faire des traductions exactes en prose. Et c'est aussi l'époque où le vocabulaire de la langue grecque moderne écrite ${ }^{7}$, qu' elle soit savante ou «vulgaire», commence à se constituer afin de désigner de nouveaux objets et de rendre de nouvelles notions.

Les textes qui pourraient faire l'objet de ce genre d'études sont assez peu nombreux et datent principalement de la fin du siècle. On peut penser à la traduction des Voyages du jeune Anacharsis de l'abbé Barthélémy par divers auteurs dont Rhigas ${ }^{8}$, aux Voyages de Télémaque de Fénelon ${ }^{9}$, aux Entretiens sur la pluralité des mondes de Fontenelle par Panayotis Kodrikas ${ }^{10}$, à la Véritable politique des personnes de qualité, ouvrage anonyme traduit par Christodoulos Pablékis ${ }^{11}$ et aux six nouvelles des Contemporaines de Restif de la Bretonne adaptées par Rhigas dans l'École des amants délicats ${ }^{12}$.

Le premier objet de ce genre d'études de traductologie historique 
devrait être de déterminer les raisons du choix du texte français par le traducteur grec. A cette époque, les traducteurs grecs, qui étaient animés de l'idéal des Encyclopédistes français, se sont d'abord proposé l'instruction de leurs lecteurs. Cela les a amenés à donner en grec des textes de vulgarisation scientifique comme les Entretiens de Fontenelle ou le Florilège de Physique excerpté par Rhigas ${ }^{13}$, principalement à partir de l'Encyclopédie. Mais la diffusion des Lumières avait aussi des objectifs religieux et moraux. Il s'agissait de remplacer la «superstition», c'est-à-dire la morale de la société chrétienne traditionnelle, par le culte de la raison et de la science, un vague déisme et une morale reposant sur la bienfaisance naturelle et une aspiration au bonheur terrestre tenu pour légitime ${ }^{1 / 1}$. L'ensemble de ce programme ne correspondait pas toujours à ce que souhaitaient les Grecs à la veille de leur indépendance ${ }^{15}$. C' est pourquoi la plupart des traductions du français visent principalement à répandre les connaissances scientifiques, plutôt que la nouvelle «morale» venue de Paris. Il reste que les Grecs n'ont pas totalement ignoré les théories pré-révolutionnaires les plus radicales.

G'est dans ce sens que la traduction, à Vienne en $1797^{16}$, par Stéphanos Dimitriadis de Skiathos ${ }^{17}$, de larges extraits de L'An deux mille quatre cent quarante. Rêve s'il en fut jamais (1770) de Louis-Sébastien Mercier (1740-1801) prend toute sa signification. Cet ouvrage de science-fiction présente comme réalisé dans un lointain avenir tout le programme révolutionnaire des Philosophes; il fut interdit en France au moment de sa parution, mais connut par la suite un succès constant puisqu'il fut réédité régulièrement tous les ans jusqu'à la fin du siècle.

La traduction de Stéphanos Dimitriadis se trouve être, à notre connaissance, le premier ouvrage de science-fiction publié en grec depuis l'Icaroménippe et l'Histoire vraie de Lucien ${ }^{18}$. Bien que Dimitriadis indique très nettement que son livre est traduit «d'un livre en langue étrangère»

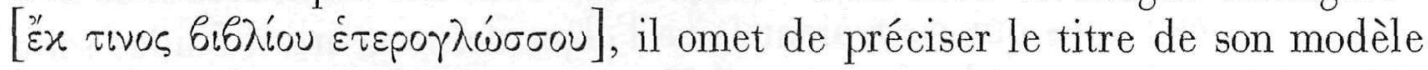
français et le nom de son auteur. Cela a provoqué les soupçons injustifiés du dernier éditeur de l'ouvrage, qui imaginait présenter au public l'œuvre originale d'un Jules Verne grec:

«Bien qu'il soit dit dans le titre que Dimitriadis a traduit ce Florilège, il n'est pas difficile de s'apercevoir qu'il est lui-même l'auteur de cette brochure contenant des idées très audacieuses pour cette époque d'obscurantisme ; on voit aussi très nettement à certains endroits l'effort qu'il fait pour plaire à l'esprit religieux de son temps. Il feint de traduire une œuvre étrangère, peut-être pour éviter d'être poursuivi ou tourné en ridicule.. ${ }^{19}$ 
En fait, la confrontation du texte de Dimitriadis avec le roman de LouisSébastien Mercier prouve à l'évidence que l'auteur grec s'est contenté de traduire de larges extraits du romancier français ${ }^{20}$. Il est très probable que la traduction n'a pas été faite sur l'édition de 1770, récemment reprise et commentée par Raymond Trousson ${ }^{21}$, car la version grecque contient quelques développements qui ne figurent pas dans cette édition; et nous savons, par ailleurs, qu'à l'occasion des rééditions Louis-Sébastien Mercier a considérablement enrichi son ouvrage ${ }^{22}$.

\section{Le choix}

Comme il l'indique dans son titre, Stéphanos Dimitriadis a procédé à un choix. Et ce choix a été très sélectif, puisque le traducteur a ramené à 48 pages un livre qui dans l'édition de 1770 , reprise en 1971, n'en compte pas moins de 341. Le choix de Dimitriadis n'est pas un résumé de l'ensemble du livre, mais une traduction complète de larges extraits des chapitres suivants: ch. II, «J'ai sept cents ans», ch. IV, «Les portefaix», ch. V, «Les voitures», ch. VIII, «Le nouveau Paris», ch. XII, «Le Collège des QuatreNations», ch. XIV, «L'Hôtel de l'inoculation», ch. XXI, «Communion des deux infinis», ch. XXIII, «Le pain, le vin, etc.», ch. XXIV, «Le prince aubergiste», ch. XXXI, «Le cabinet du roi», ch. XL, «Du commerce», ch. $X L I$, «L'avant-souper». Stéphanos Dimitriadis n'a absolument rien emprunté aux autres chapitres, sans doute parce qu'ils traitaient plus précisément de politique, de littérature et même de modes françaises ${ }^{23}$. Les sujets qui ont été retenus comme étant de nature à intéresser les Grecs ont trait aux bâtiments, à la circulation en ville, à la médecine, à la science naturelle, à l'instruction publique, aux repas et au commerce.

\section{La transposition}

Quand on en vient à l'examen de la traduction on s'aperçoit que Dimitriadis a eu soin d'adapter son texte à la réalité du monde grec du temps et aux besoins de ses compatriotes.

A aucun moment la France du XVIIIe siècle, qui était omniprésente dans le texte de Mercier, n'apparaît dans le texte grec. Quand Mercier parle d'«un homme du siècle de Louis $X V »$, Dimitriadis mentionne vaguement

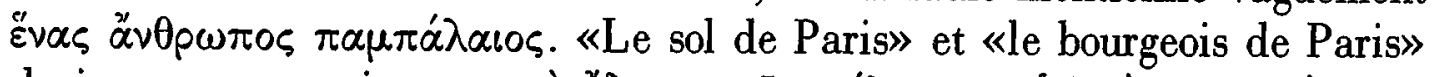

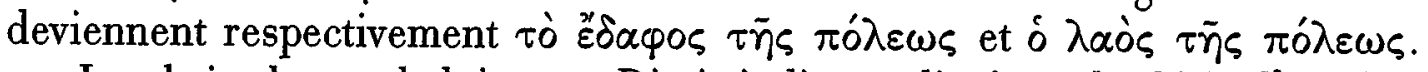

Le choix du vocabulaire par Dimitriadis est dicté par le désir d'éveiller 
des idées précises chez un sujet grec de l'empire ottoman, en particulier à Constantinople et dans les Principautés danubiennes. Ainsi, ayant à traduire «Monsieur, le fils de la maison», l'auteur grec trouve un équivalent très évo-

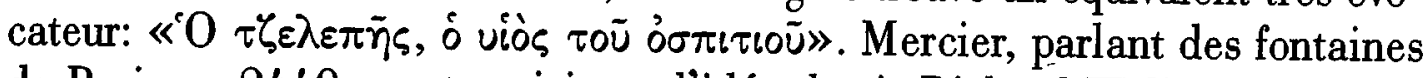
de Paris en 2440, - et anticipant l'idée de sir Richard Wallace-, notait que chacun pouvait $\mathrm{y}$ boire et, faisant allusion à leur forme, remarquait: «Cette coquille présentait à chaque passant une tasse salutaire.» Dimitriadis a imaginé cela à l'image des fontaines turques munies d'un gobelet métallique attaché à une chaîne et à la disposition de chacun: عís xáa $\theta \varepsilon$ bpú-

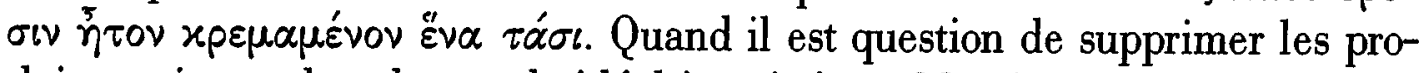
duits excitants dans le monde idéal imaginé par Mercier, Dimitriadis donne une certaine couleur locale à son texte en ajoutant le raki à la liste des produits prohibés ${ }^{2 / 1}$. Comme les spécialités culinaires françaises sont très variées et n'ont rien à voir avec la cuisine turque, Dimitriadis s'en tire comme il peut en traduisant «ragoût, jus, coulis» par un seul mot « $\pi \varepsilon \lambda \tau \varepsilon \dot{\varepsilon}$ ».

De même les problèmes de l'éducation en France ne pouvaient guère être rapprochés de ceux d'une Grèce qui n'existait pas encore comme Etat indépendant et ne possédait pas de langue vernaculaire écrite unifiée. Gependant Dimitriadis a su transposer la prééminence du français sur le latin en parlant du remplacement du grec ancien par la langue parlée, appelée langue «simple». D'où la traduction de la phrase de Mercier: «La

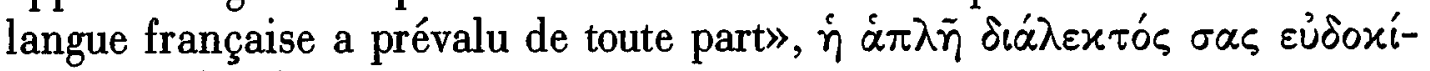
$\mu \eta \eta \sigma \varepsilon \varkappa \alpha \tau \grave{\alpha} \pi \alpha ́ v \tau \alpha$.

\section{L'effacement des détails intraduisibles}

Dimitriadis cherchait avant tout à produire un texte lisible par des Grecs, texte qui, après tout, aurait très bien pu être une æuvre grecque originale. Mais il s'est heurté à beaucoup de difficultés tenant à la grande différence existant alors entre le français et le grec vulgaire. Alors que le vocabulaire du français n'avait cessé de s'enrichir et de se préciser depuis au moins le XVIe siècle, Dimitriadis devait effectuer sa traduction dans une langue simple ( $\left.\dot{\alpha} \pi \lambda \bar{\eta} \delta \iota^{\prime} \alpha \lambda_{\varepsilon x \tau \sigma \varsigma}\right)$ qui n'était pas encore fixée et dont le vocabulaire dans le domaine des realia et des notions modernes était d'une insigne pauvreté. Quand on prend conscience de cela, on ne peut qu'être admiratif devant le travail du traducteur grec. Même lorsqu'il esquive les difficultés - et cela est fréquent-, il le fait avec beaucoup d'intelligence et parfois d'élégance. Ainsi, faute de disposer d'un mot pour

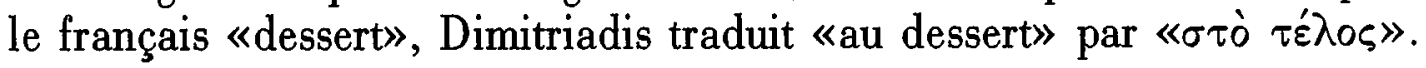


N'ayant rien de satisfaisant pour les mots «pavé» et «affiche» il s'en tire en utilisant les mots $\varepsilon^{\prime} \delta \alpha \varphi \circ \varsigma_{\text {e }}$ et $\varepsilon^{\prime} \gamma \gamma \rho \alpha \varphi \circ$. Dans ces cas nous avons simplement une banalisation de la notion mais sans trahison grave du sens.

Comme la plupart des Grecs non Constantinopolitains habitaient alors dans des agglomérations de taille réduite, le grec «vulgaire» manquait de mots pour désigner les réalités de la grande ville. Ainsi, ayant à traduire

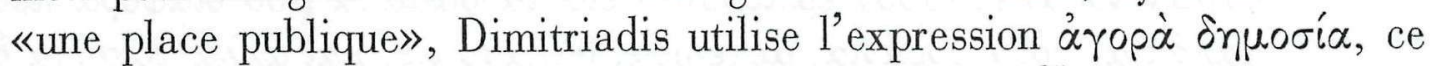
qui pour nous évoque plutôt un lieu de la cité antique ${ }^{25}$. Il traduit «belles rues» par $\omega$ paias $\pi \lambda \alpha \tau \varepsilon i \alpha s$, ce qui confirme que les équivalents actuels pour «rue» et «place»n’étaient alors ni précis ni différenciés.

Dimitriadis a parfois, faute de mieux, recours à des périphrases, et il faut reconnaître que certaines sont fort bien venues. Ayant à traduire «bâtiments élégants», ce qui aujourd'hui ne poserait pas de problème, il opte

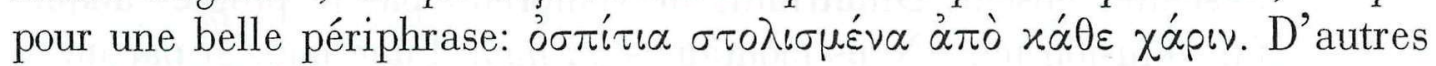
fois, la réalité française à traduire étant encore inconnue en Grèce, il a recours à une sorte de définition de dictionnaire ${ }^{26}$, ce qui peut être supportable si celle-ci n'est pas trop longue. Ainsi pour «observatoire» ${ }^{27}$ qui

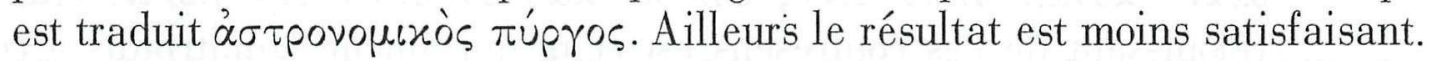
C'est le cas pour le mot «bouchon» (sorte de restaurant) qui fait difficulté même pour un lecteur français d'aujourd'hui: «Je traversai plusieurs rues sans rencontrer un seul bouchon». Dimitriadis, qui a sûrement consulté un dictionnaire de définitions français, aboutit à la traduction suivante qui ne rend pas le sens propre du mot français: «Je traversai plusieurs rues sans rencontrer aucune enseigne de celles que l'on trouve devant les portes des

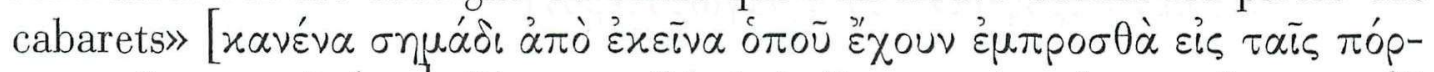

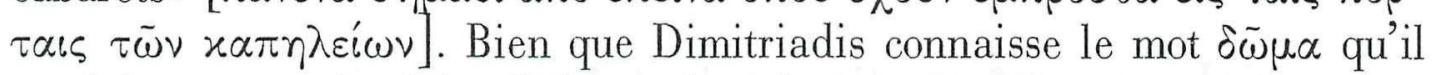
emploie un peu plus loin, il donne dans le passage suivant une interprétation étymologique du français «terrasse» particulièrement incongrue dans

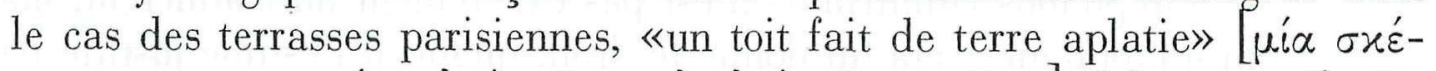

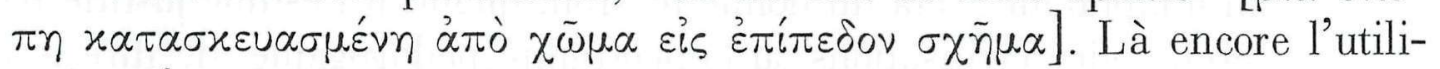
sation d'un dictionnaire français est visible.

\section{Les faux-sens et les contresens}

L'étude scientifique d'une ancienne traduction ne doit pas éluder l'examen des fautes, ou de ce qui nous apparaît comme tel, afin de mettre en évidence les différences de civilisations que la traduction tente d'annuler.

Les fautes de traduction les plus fréquentes sont les omissions injustifiées et les mauvaises lectures de l'original. Soit le passage suivant de 
Mercier: «On fit passer sous mes yeux des paysages, des points de vue, des palais, des arcs en ciel, des météores, des chiffres lumineux, des mets qui n'existaient point.» Embarrassé par certaines expressions qu'il ne comprenait pas - et, à ce point de vue, l'absence d'équivalent pour "paysage» ${ }^{28}$ est caractéristique - Dimitriadis a pris le parti de ne pas les traduire: " $\mathrm{E}$ -

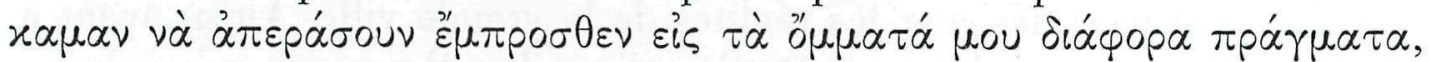

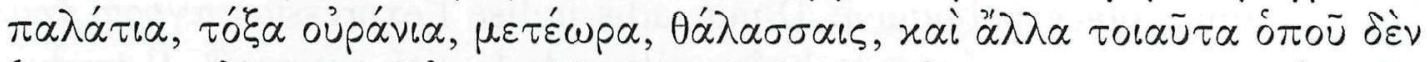

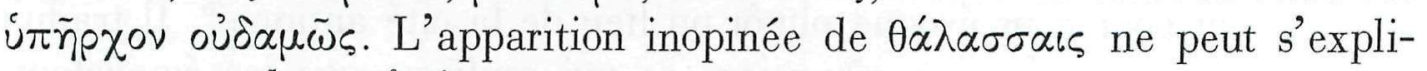
quer que par la confusion entre «mets « et «mers».

Certaines inexactitudes proviennent de la méconnaissance de la réalité quotidienne du pays dans lequel se déroule l'œuvre que l'on traduit. $\mathrm{N}^{\prime}$ ayant pas vécu dans une maison parisienne aux nombreux étages et à l'escalier obscur, Dimitriadis ne comprend pas le progrès auquel Mercier fait allusion ici: «Nous montâmes au haut d'une maison par un escalier où l'on voyait clair.» Le traducteur grec croit que cela signifie que l'on a une

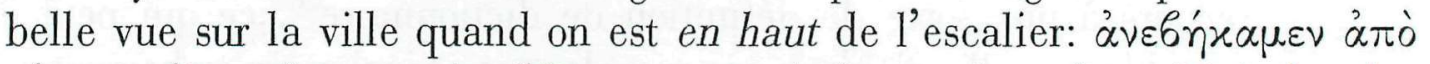

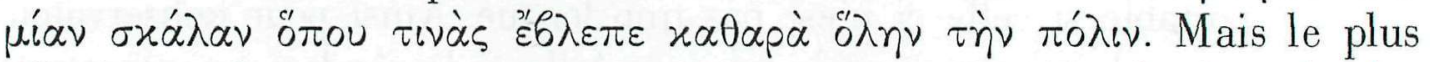
réjouissant de ces contresens est celui qui amène Dimitriadis à confondre la portière d'un carrosse avec la femme du portier. Mercier écrivait à propos d'un riche Parisien pris dans un encombrement: «il criait à la portière et se lamentait de ne pouvoir avancer.» Le traducteur grec entend cela bien différemment: «il appelait la portière à grands cris et se plaignait

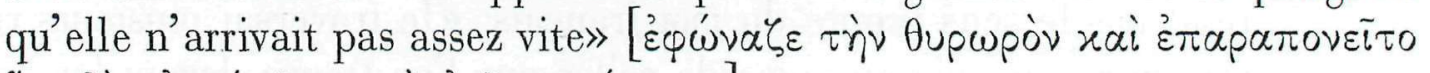

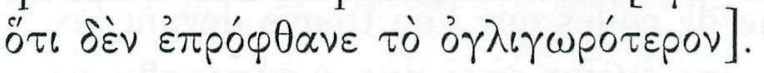

\section{Les transformations volontaires}

Stéphanos Dimitriadis n'est pas exactement un traducteur, mais plutôt un adaptateur. Cela lui donne le droit, quand il l'estime justifié par la différence des civilisations ou l'opportunité pédagogique, de dire le contraire de ce qu'il trouve dans son modèle.

Ainsi à propos des manières de table, Mercier précisait que la meilleure éducation présidait aux repas d'où les excès de la gourmandise étaient bannis: «chacun se servait et avait sa portion vis-à-vis de soi. Celui-là eût passé pour gourmand qui aurait été au-delà de sa portion: elle était suffisante.» Dimitriadis s'en prend à un autre excès, bien réel en Grèce, celui des hôtes qui, pour donner, à ce qu'ils croient, une bonne idée de l'abondance qui règne chez eux, s’ingénient à rassasier leurs invités ... même audelà de ce qu'ils peuvent supporter, et contre leur volonté. «Chacun se ser-

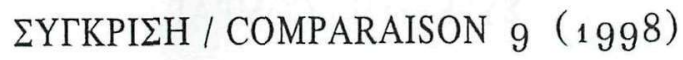


vait tout seul, sans qu'on lui remplisse son assiette de nourriture qu'il ne pouvait manger. Il en prenait autant qu'il en voulait et en mangeait juste ce qui lui suffisait sans surcharger son estomac pour faire plaisir à ceux qui le servaient surabondamment. $»^{29}$

Il arrive aussi, mais rarement, que Dimitriadis modifie profondément son modèle, parce qu'il n'est pas d'accord avec la thèse soutenue par Mercier. C'est le cas à propos de l'enseignement de l'histoire que nous qualifierions d'événementielle et que Mercier voudrait voir disparaître des programmes, parce qu' elle donne surtout des exemples de la conduite immorale des souverains, alors que Dimitriadis y aperçoit plutôt des manifestations de la gloire passée des Grecs. Mercier s'exprime en ces termes: «0n leur enseigne peu d'histoire, parce que l'histoire est la honte de l'humanité, et que chaque page est un tissu de crimes et de folies.» Dimitriadis a tourné cela de la façon la plus positive: «Ensuite, nous leur enseignons l'histoire, non point celle qui est pleine de mensonges, mais celle qui a été vérifiée par des auteurs savants et vénérables.» ${ }^{30}$ La mếme tendance à tourner en préceptes positifs ce que Mercier écrivait de façon polémique et révolutionnaire s'observe à propos de la langue. Mercier affirmait que chaque génération a le droit imprescriptible de modifier à sa guise la langue nationale. L'auteur grec, dont les problèmes linguistiques sont tout différents, ne veut voir dans cette liberté que la possibilité d'une collaboration féconde entre la jeunesse et les doctes pour l'amélioration de la langue nationale. Mercier écrivait: «Nous leur enseignons la langue nationale, et nous leur permettons même de la modifier d'après leur génie, parce que nous ne voulons pas des grammairiens mais des hommes éloquents.» ${ }^{31}$ Dimitriadis conserve les mêmes expressions mais délivre un autre message: «Nous nous contentons de leur enseigner la langue de notre nation; nous faisons en sorte qu'ils y appliquent leurs soins et leur suggérons de l'amener à plus de clarté, en fonction de leur génie, car nous ne voulons pas de grammairiens obscurs mais éloquents.» $\mathrm{On}$ n'est pas loin ici des idées de Coray.

\section{Les réussites de la traduction}

Malgré toutes ces observations, il ne faudrait pas croire que la traduction de Stéphanos Dimitriadis soit médiocre. Ce petit ouvrage se lit avec agrément pour plusieurs raisons.

En premier lieu Dimitriadis a su purger le texte de Louis-Sébastien Mercier de toute la verbosité qui en est le principal défaut. Ensuite la «langue simple» qu'il emploie a beaucoup de charmes. Elle est dénuée des lourdeurs de celle de Katardzis, des archaïsmes de la langue mixte de 
Coray et des nombreux xénismes ${ }^{32}$ de la prose phanariote telle qu'on peut la lire par exemple dans les Ephémérides de P. Kodrikas ${ }^{33}$.

L'auteur grec a rendu un certain équilibre au style parfois heurté de Mercier. Il lui est arrivé par exemple de réunir en une période deux phrases courtes de son modèle: «Le soleil baissait. Mon guide me sollicita d'en-

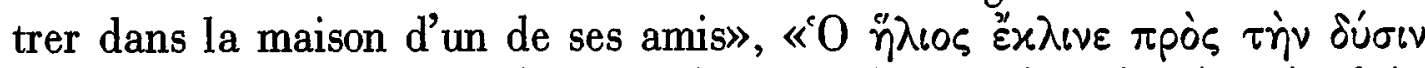

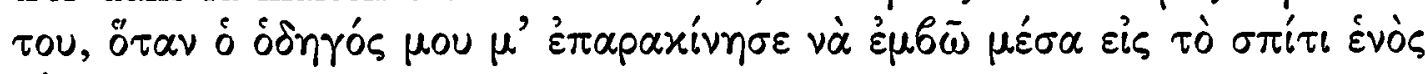

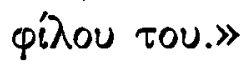

Là où Mercier suggère, Dimitriadis développe un ensemble cohérent. Ainsi dans l'opposition suivante entre la jeunesse et la vieillesse: «En me regardant dans un miroir j'eus peine à reconnaître mon visage. Je m'étais couché avec les cheveux blonds, mon front était sillonné de rides, mes cheveux avaient blanchi, javais deux os saillants au-dessous des yeux, un long nez et une couleur pâle et blême était répandue sur toute ma figure.» Cela devient chez Dimitriadis un parallèle parfait [nous écrivons en italiques les ajouts et modifications du traducteur]: «Quand je m’étais couché j’avais des cheveux blonds, la peau blanche et du rouge aux joues; mais lorsque je regardai mon visage dans le miroir, il était tout plein de rides; mes cheveux étaient blancs et j’avais deux os saillants au-dessus des yeux, un nez long et le teint pâle.» ${ }^{3 / 4}$ On remarquera que si Mercier tient la pâleur pour un défaut de la vieillesse, Dimitriadis tient la blancheur du teint pour un attribut de la jeunesse. Le traducteur tient aussi compte des canons de beauté de son pays ... Quant aux os saillants l'auteur grec imagine qu'il s'agit des arcades sourcilières, ce qui les lui fait placer au-dessus des yeux.

Il arrive même à Dimitriadis de réussir une chose difficile dans la traduction, rendre les nuances du modèle par une variation du niveau de langue. Dans un passage à caractère philosophique l'auteur grec rend la solennité d'une sentence en donnant une couleur biblique à son grec. Mercier exprimait la ferveur de sa foi déiste et finaliste, polémiquant peut-être de façon indirecte contre Voltaire. «On dit sous l'œil de Dieu: Tout est bien.» Pour rendre la solennité du ton Dimitriadis emprunte à juste titre

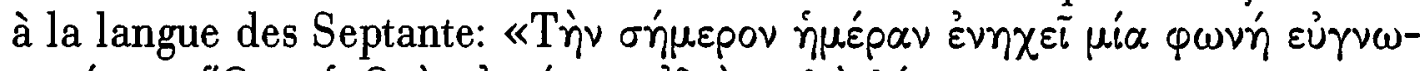

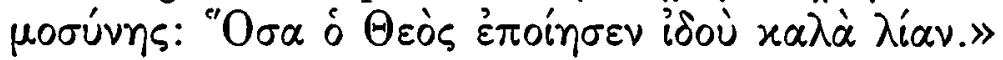

\section{Conclusion}

L'histoire des idées, des courants littéraires et de la langue grecque aurait beaucoup a gagner à l'étude systématique des plus anciennes traductions. On peut y voir se créer les nouvelles notions et les mots qui les 
expriment. De telles études montreraient les progrès considérables accomplis par le vocabulaire néo-hellénique en deux siècles. Et cela aiderait à constituer un dictionnaire historique du grec moderne du XVIII siècle à la fin du XXe siècle, instrument qui fait encore défaut à nos études.

\section{$\mathrm{Notes}$}

1. Sur ce sujet, lire en général Constantin Th. Dimaras, La Grèce au temps des Lumières, Genève, 1969 et en grec, du

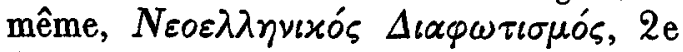
éd., 1980, Paschalis M.Kitromilidès,

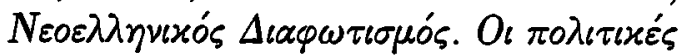

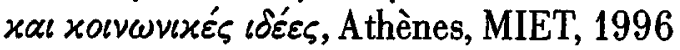

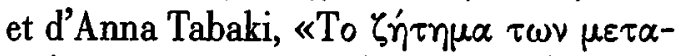

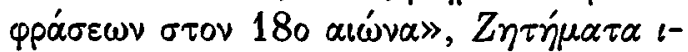

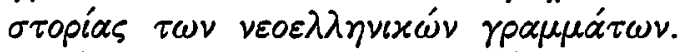

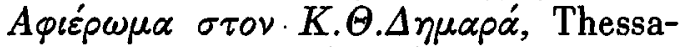
lonique, Paratiritis, (1994), 89-106.

2. Parmi les ouvrages consacrés à ce sujet on distinguera le livre commode de

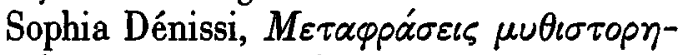

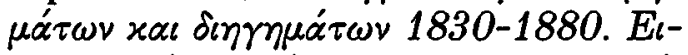

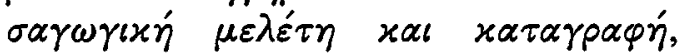
Athènes, 1995. Pour le XVIIIe siècle, si l'on veut disposer d'une liste complète et de beaucoup plus de renseignements, on devra consulter l'ouvrage du KNE/

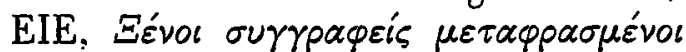

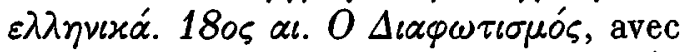
une introduction d'Anna Tabaki (à paraitre). L'ouvrage complet comprendra 3 volumes.

3. J'ai tenté une étude de ce type

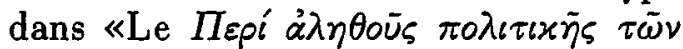
$\varepsilon \dot{\gamma} \gamma \varepsilon v \tilde{\omega} \nu$ í $\pi \circ x \varepsilon \mu \varepsilon^{\prime} v \omega \nu$ (1781). Identification de la source française. Premières

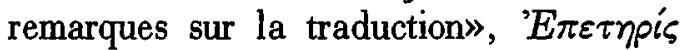

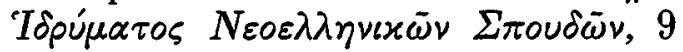
(1995-1996), 187-192

4. Citons, de façon indicative, le cas

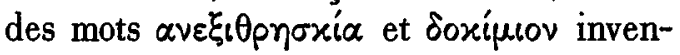
tés en 1768 par Eugène Voulgaris pour rendre les termes «tolérance» et «essai» de Voltaire et celui de $\chi a \mu i v t$, simple retran- scription du français «gamin» que l'on doit à Isidoridis Skylitsis dans sa traduction des Misérables de Victor Hugo à Vienne en $1862\left(\mathrm{n}^{\circ} 300 \mathrm{du}\right.$ catalogue de Dénissi); voir sur ce dernier point l'article de l' $E$ -

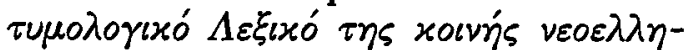
$v e x \eta^{2} \zeta^{2} 1967$ de N. P. Andriotis. Sur les traductions de Skylitsis et leur impact sur le vocabulaire grec, lire Anna Tabaki, « $\mathrm{O}_{\iota} \varepsilon x-$

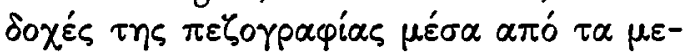

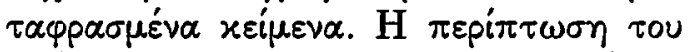

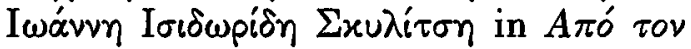

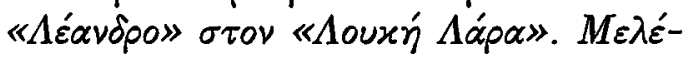

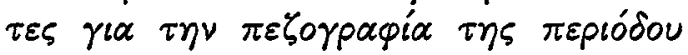
1830-1880, pp. 191-204.

5. Bien que Byzance ait vécu dans un état de quasi-autonomie culturelle, il vint un moment où la traduction de certains textes religieux ou philosophiques latins en grec devint indispensable, ne serait-ce que pour les nécessités de la controverse. On peut, dans ce domaine, citer des traductions de Saint Augustin et de Saint Thomas d'Aquin en grec byzantin, c'est-à-dire en grec ancien, par Maxime Planude et les frères Kydonès ; voir là-dessus par exem-

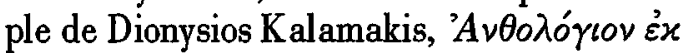

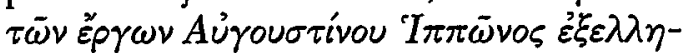

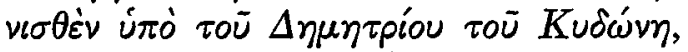
Athènes, 1996 et d'une façon plus générale, sur les contacts entre le monde byzantin et post-byzantin et l'Occident, la monographie de Deno J. Geanakoplos (trad.

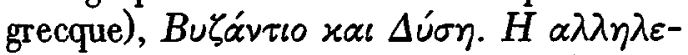

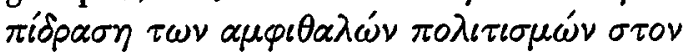

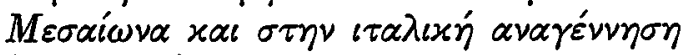
(330-1600), Athènes, 1985. Le Moyen Age a aussi connu quelques traductions de 
romans occidentaux, comme le Roman de Troie de Benoît de Sainte Maure, récemment réédité à partir des manuscrits par $\mathrm{M}$. Papathomopoulos et E.M. Jeffreys, O $\Pi{ }^{\prime}-$

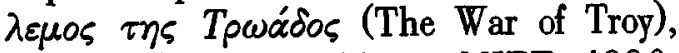

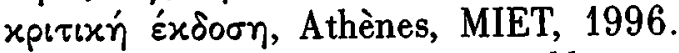
Cette édition tout à fait remarquable pour l'établissement du texte grec reste assez malcommode pour l'étude de la traduction puisque le texte français (ou du moins une de ses versions) n'a pas été publié en regard, mais seulement par bribes dans l'apparat critique. Le tableau comparatif présenté aux pp. LII et LIII de l'Introduction montre qu'à peu près la moitié du texte français n'est pas traduite et que le reste est rendu de façon très «générale».

6. Sur la traduction au XVIIIe siècle,

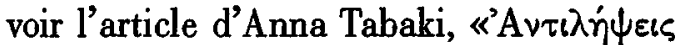

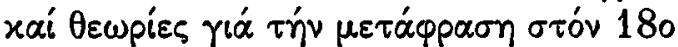

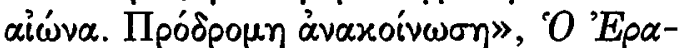

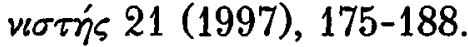

7. Sur l'histoire de la langue grecque moderne écrite, voir notre communication «Note sur le grec écrit de la fin de l'antiquité à la fin du XVIIIe siècle» in $\mathrm{La}$ Grèce moderne et l'héritage du passé, textes réunis par Renée Richer, Nice, Centre Universitaire d'Etudes Grecques Modernes, 1997, pp. 98-112.

8. Ces traducteurs sont Constantin Sakellarios de Kozani, Georges Vendotis de

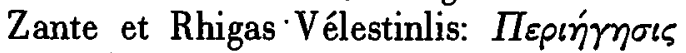

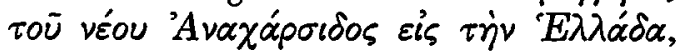

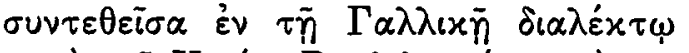

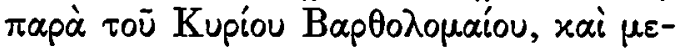

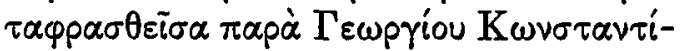

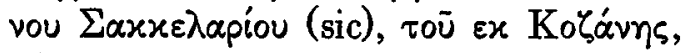
Vienne 1797. Le vol. IV est traduit par Rhigas et Vendotis sous le titre NÉos Avá-

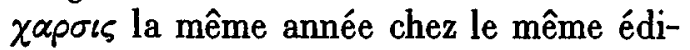
teur (Vendotis). Sur les traductions de Rhigas lire spécialement d'Anna Tabaki, «To

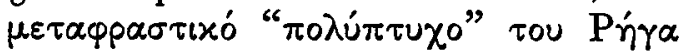

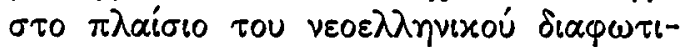

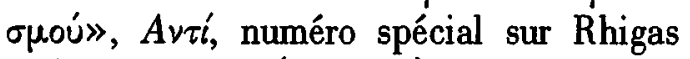
Vélestinlis, 652 (16/1/98), 39-43.

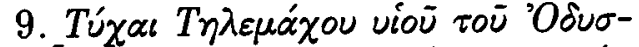

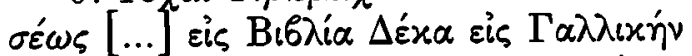

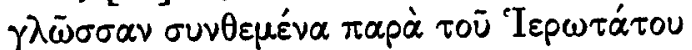

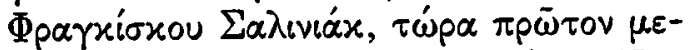

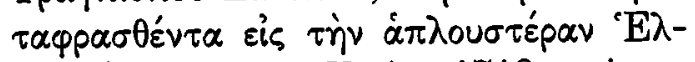
$\lambda \eta \nu เ x \dot{v} v \Gamma \bar{\omega} \sigma \sigma \alpha v$, Venise, 1742 ; voir sur cette traduction, Émile Legrand, Bibliographie Hellénique (XVIIIe siècle), 1918, vol. I, pp. 301-302 et maintenant

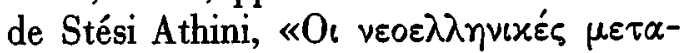

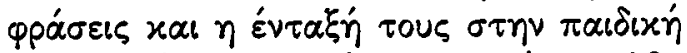

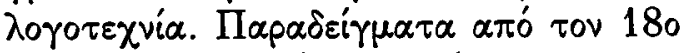

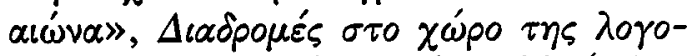

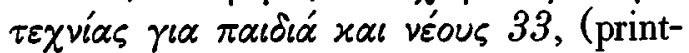
emps 1994), 30-38.

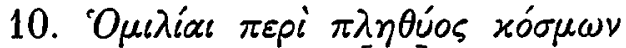

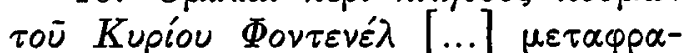

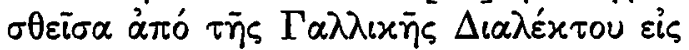

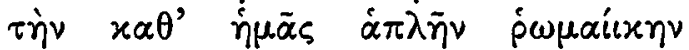

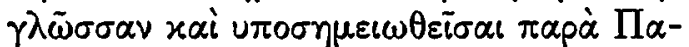

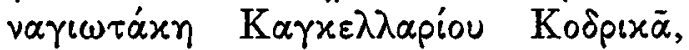
Vienne, 1794.

11. Sur cette traduction, voir notre

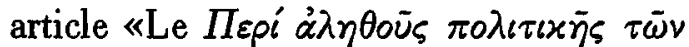

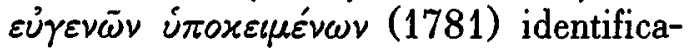
tion de la source française. Premières remarques sur la traduction» cité plus haut.

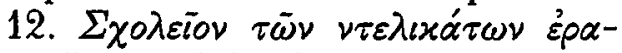

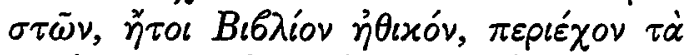

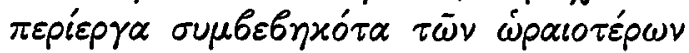

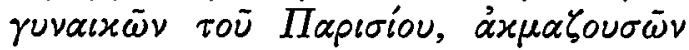

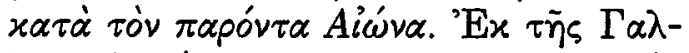

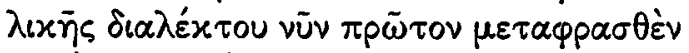

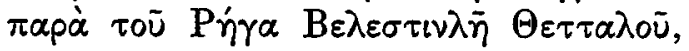
Vienne 1790. Edition récente par P.S. Pis-

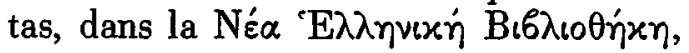
Athènes 1971 et réimp. 1994. Pour l'adaptation Athènes, française voir Yvonne Gineste, «Rhigas Vélestinlis traducteur de Restif de la Bretonne», Cahiers Balkaniques 24 (1996), 165-189.

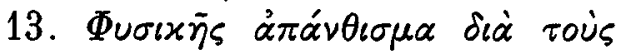

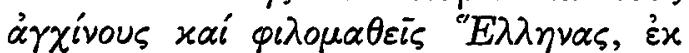

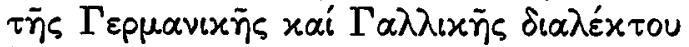

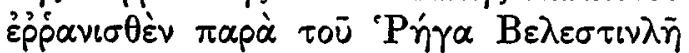
$\Theta \varepsilon \tau \tau \alpha \lambda \circ \tilde{u}$, Vienne 1790. Réédition photomécanique par la Société scientifique 
d'Etude de Phères, de Vélestino et de Rhigas, Athènes 1991. Pour la détermination des sources voir l'intéressant article de D. Karambéropoulos, «Rhigas Vélestinlis -

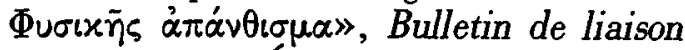
du Centre d'Études Balkaniques 12 (1994), 129-139.

14. Sur la morale de ce temps et les manuels d'éthique grecs voir Anna TabakiAlexandra Sfini, «Typologie des manuels d'éthique et de comportement en langue grecque vers la fin du XVIIIle siècle: L'évolution du genre, reflet du processus de modernisation du Sud-Est européen», RESEE, XXX (1992), nos 3-4, 253-268,

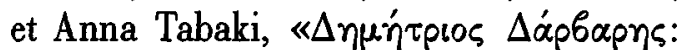

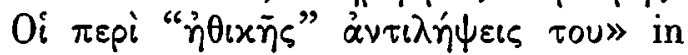

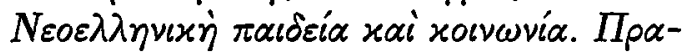

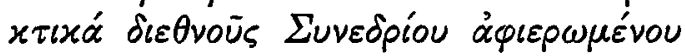
$\sigma \tau \dot{\eta} \mu \nu \eta \dot{\mu} \eta \bar{\eta}$ K. $\Theta . \Delta \eta \mu \alpha \rho \bar{\alpha}$, Athènes, 1995, pp. 107-120.

15. Voir par exemple les réactions aux idées de Rhigas que l'on peut trouver dans Michel Perdikaris (1766-1838) Rhigas ou contre les faux amis des Grecs ['Pท́ras $\ddot{\eta}$

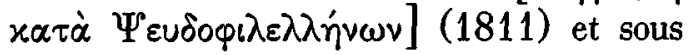
une forme moins violente mais tout aussi déterminée dans les Résultats de l'amour

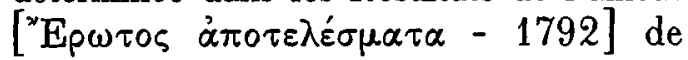
Ioannis Karatzas.

16. Le titre de ce petit livre de 48

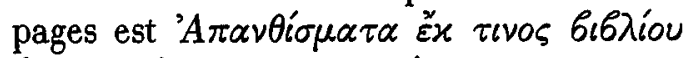

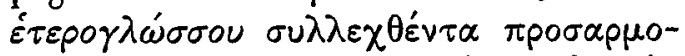

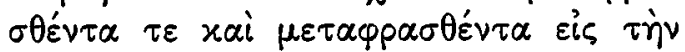

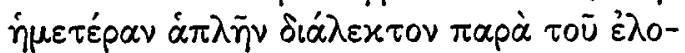

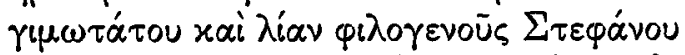

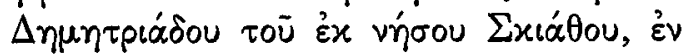

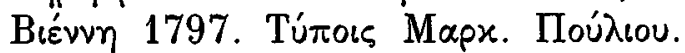
Récente réédition photomécanique aux éd. galerie «Koultoura», Athènes 1977.

17. Sur ce personnage voir Lada-

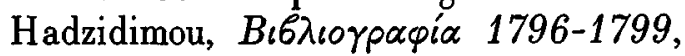
pp. 54-55, n 49. Né à Skiathos en 1760 , il mourut dans son île natale, dont il avait été voevode, en 1827. Elève de Néophytos Kafsokalyvitis, il fut en Valachie secrétaire du prince Nicolas Karatzas puis, aux côtés de Rhigas, deuxième secrétaire du prince Nicolas Mavrogénis. Il avait participé en 1821 au siège de Tripolitza.

18. La différence majeure entre ces ouvrages antiques et celui de LouisSébastien Mercier réside dans le fait que Lucien pratique l'utopie, alors que l'écrivain français se livre à une «uchronie». Sur ce genre dans les lettres grecques on peut lire notre article «Aux origines de la (science) fiction grecque: le voyage extraordinaire», Cahiers balkaniques 18 (1992), 81-100.

19. Ce texte extrait de l'introduction de l'édition de 1977 est signé $\Lambda . K .:$ «Av

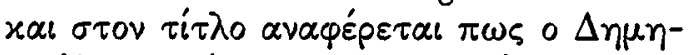

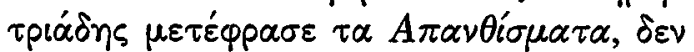

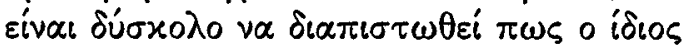

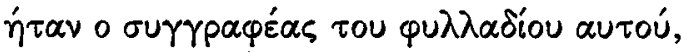

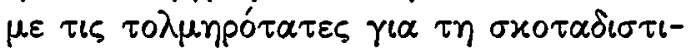

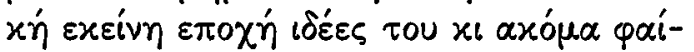

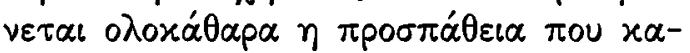

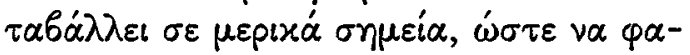

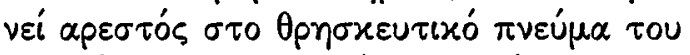

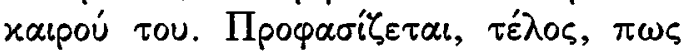

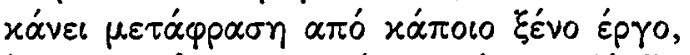

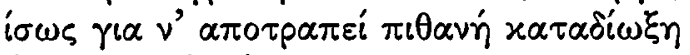

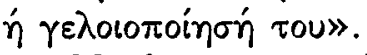

20. Sur cette traduction, lire l'étude très riche de Stési Athini, $\ll \Sigma \tau . \Delta \eta \mu \eta \tau$ plá-

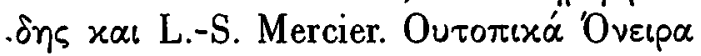

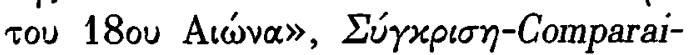
son, 6 (juin 1995), 25-39. Cet article recoupe le nôtre sur bien des points. L'identification de la source a été faite par un anonyme au ${ }^{\circ} 1$ de la revue $\Pi a ́ \lambda_{b}$ (1965), 92-95 (renseignement communiqué par Stési Athini) puis, indépendamment semble-t-il, par Makis Panorios dans un article paru dans la revue grecque Aıv' $\gamma \mu \alpha \tau \alpha$

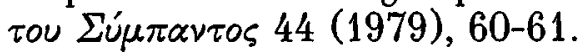

21. L'An deur mille quatre cent quarante: Rêve s'il en fut jamais, Edition, introduction et notes par Raymond Trousson, Bordeaux, éd. Ducros, 1971.

22. Le livre de L.-S. Mercier fut réédité en 1771, 1772, 1773, 1774, 
$1775,1776,1785,1786,1787,1793$, 1799. L'éditeur récent de l'ouvrage, Raymond Trousson, est très critique sur les suppléments apportés par Mercier au fil des rééditions: «Dans les versions suivantes, Mercier, incapable de rien retrancher, procède par «bourgeonnement», par greffes successives, sans s'inquiéter d'édifier un ensemble curieusement composite.» (Introduction, p.72). Par rapport à l'éd. de 1770, la traduction de Dimitriadis comprend deux paragraphes supplémentaires,

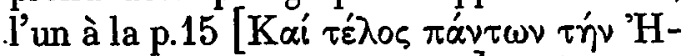

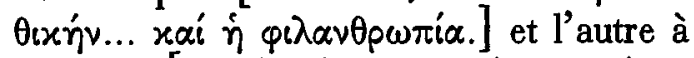

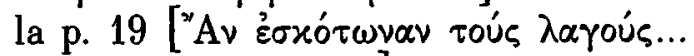

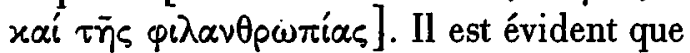
Dimitriadis a utilisé une des éditions augmentées postérieures à 1770, à l'exception, bien sûr, de celle de 1797. Une recherche dans ces éditions pourrait préciser la version utilisée par le traducteur grec.

23. On peut en juger par les titres de certains de ces chapitres omis: «Les chapeaux brodés», «Théologie et jurisprudence», «La bibliothèque $d u$ roi», «Les gens de lettres», «L'Académie française», «De l'héritier du trône», «Versailles».

24. «Nous avons sagement banni trois poisons physiques dont nous faisions un perpétuel usage: le tabac, le café et le thé». Chez Dimitriadis la prohibition concerne

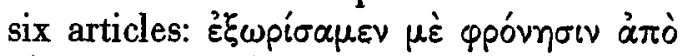

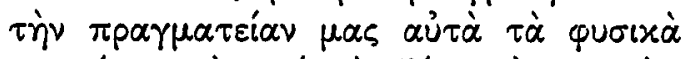

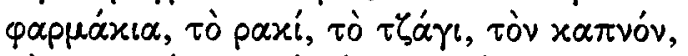

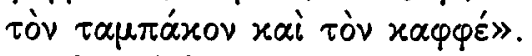

25. Il faut cependant reconnaître que le grec «ottomanisé» de l'époque disposait d'autres mots que áyopá pour désigner un

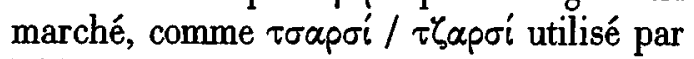
Rhigas en 1790 et Caesarios Dapontes avant 1784 .

26. Il est du reste pour moi très vraisemblable que Dimitriadis utilisait pour ses traductions un dictionnaire de définitions français et peut-être, si, comme c'est probable, il connaissait l'italien, la partie italo-grecque du Tesoro della lingua Greca- volgare du père Alexis de Somevoir (=Alessio da Somavera), Paris 1709. C'était encore le principal instrument lexicographique bilingue utilisé en 1827 par Coray pour rédiger les articles de ce qui est, à ma connaissance, le premier dictionnaire français-grec (là-dessus, voir plus bas à la note 26 ).

27. Selon Stéphanos A. Koumanoudis, $\Sigma u v a \gamma \omega \gamma \dot{n} N_{\varepsilon}^{\prime} \omega \nu \Lambda \varepsilon^{\prime} \xi \varepsilon \omega \nu, 1900$, le mot

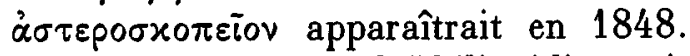
Précédemment Daniel Philippidis avait employé á $\sigma \tau p o \sigma x o ́ \pi t o v$ en 1803.

28. Koumanoudis, op. cit, n'enregistre

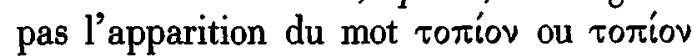

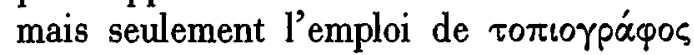
pour «paysagiste», ce qui fait supposer que le mot simple existait antérieurement. De fait, la chose est confirmée par le long article que Coray consacre au mot dans son

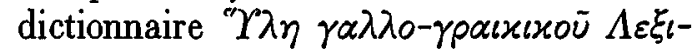
xoũ (achevé par le savant en 1827 et édité après sa mort [1833] par Andréas Mamoukas, récemment réédité par Alkis

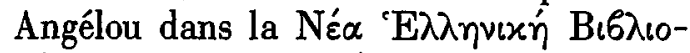
$\theta$ ñर

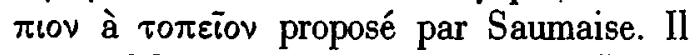
reprend la même doctrine dans les " $A \tau \alpha$ $x \tau \alpha 2,1829$, pp. 426-429 où il précise que le mot est un hellénisme de Vitruve VII,5 chez qui il désigne les paysages dessinés. «C'est par le mot tótıov donc que nous devons traduire chaque fois que cela est nécessaire, le mot «paysage» des Français.» Mais le mot proposé par Coray a eu du mal à s'imposer; en 1883 Vizyinos emploie encore $\chi \omega \rho$ proppa $\varphi^{\prime} \alpha$ dans ce sens (recensé par Koumanoudis comme un mot de Vizyinos, mais datant de 1895).

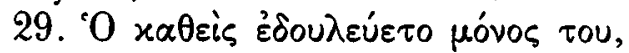

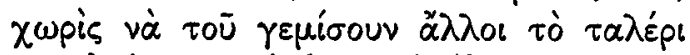

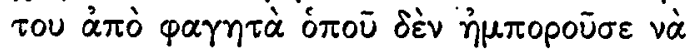

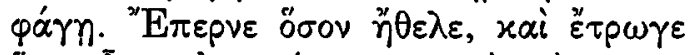

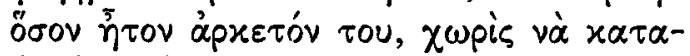

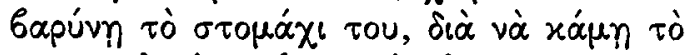

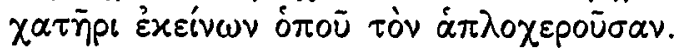

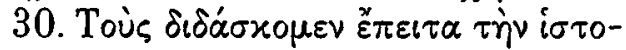




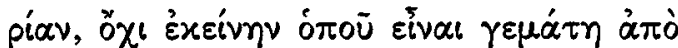

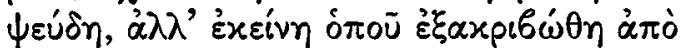

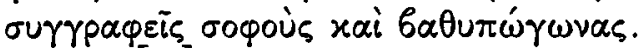

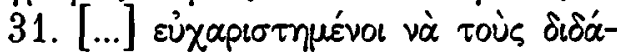

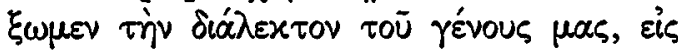

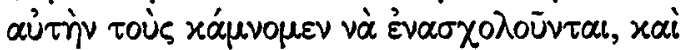

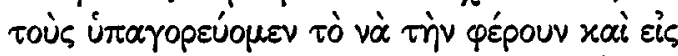

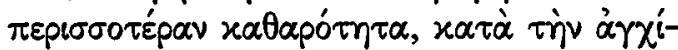

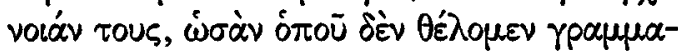

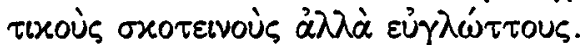

32. On relève dans le texte de Dimitriadis les turcismes suivants: $\tau \dot{\alpha} \sigma \iota, \pi \alpha \zeta^{\prime} \alpha-$

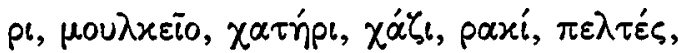

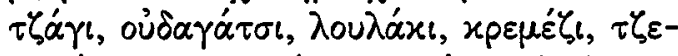

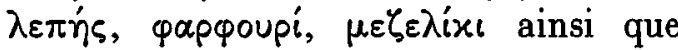

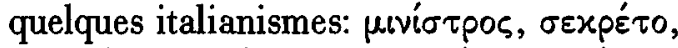

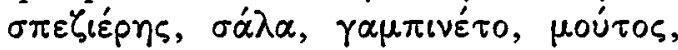

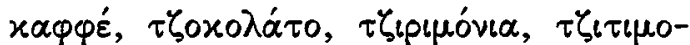
vió̧os et quelques emprunts français: $x \lambda \dot{\alpha}-$

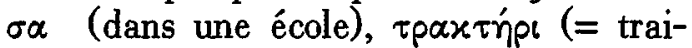

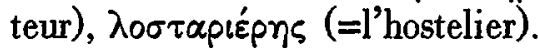

33. On trouvera une édition de ce texte intéressant tant du point de vue du contenu que de celui de la langue par Alkis

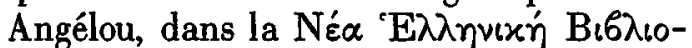
Өńxy de la maison Ermis, Athènes, 1991.

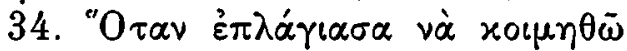

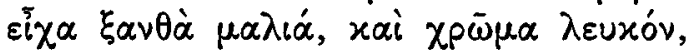

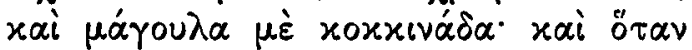

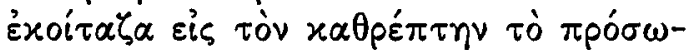

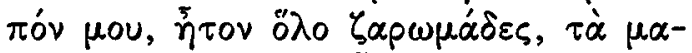

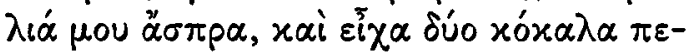

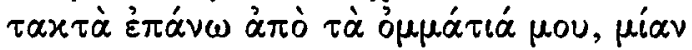

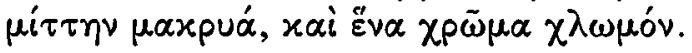

\section{$\Pi \varepsilon \rho i \lambda \eta \psi \eta$}

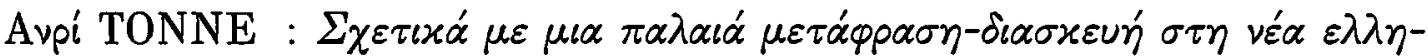
vเxý tou Lous-Sébastian Mercier

$\mathrm{T}$

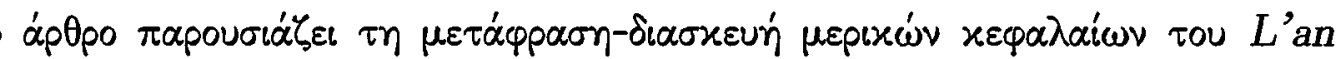

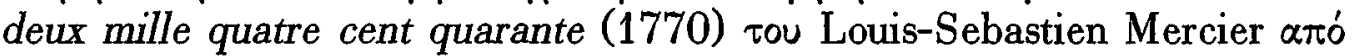

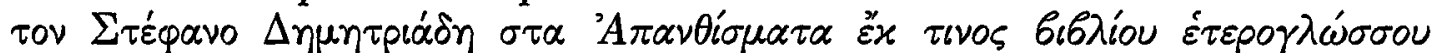

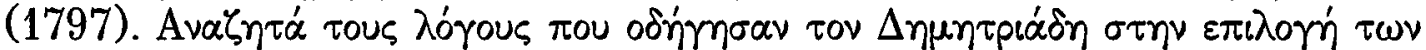

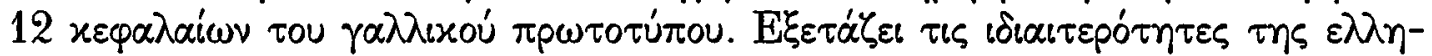

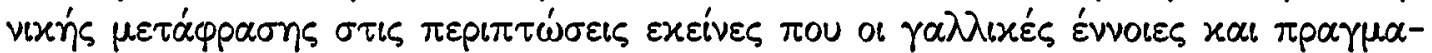

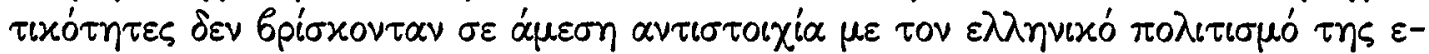

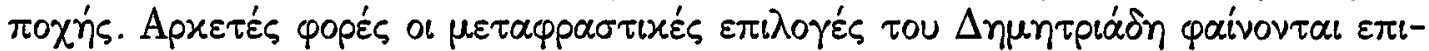

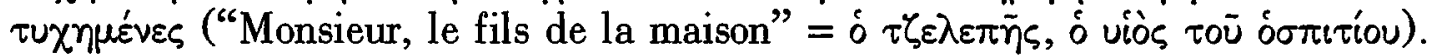

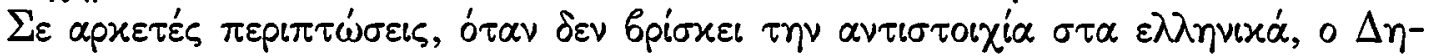

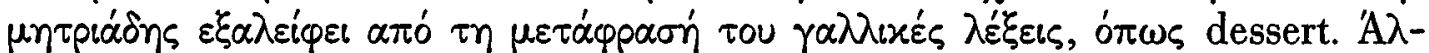

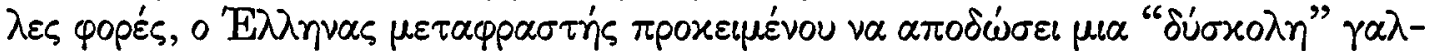

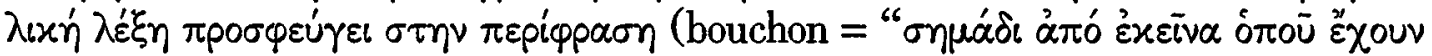

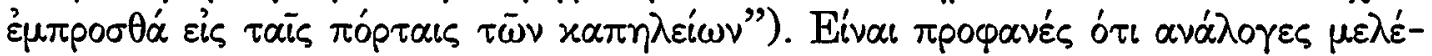

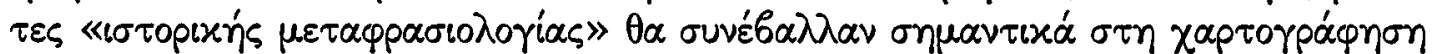

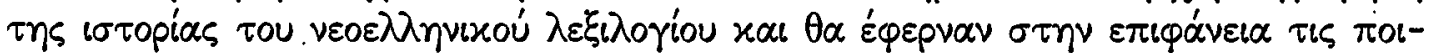

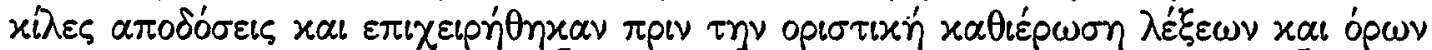

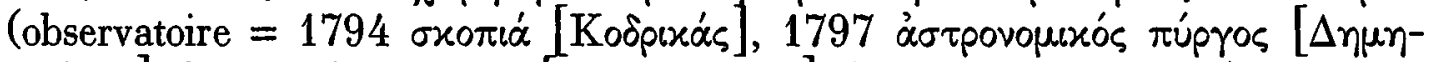

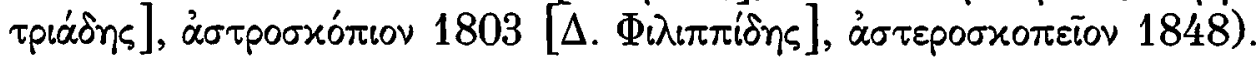

